\title{
Toward Optimization of Centrifugal Barrel Polishing Procedure for Treatment of Niobium Cavities
}

\author{
Alena Prudnikava, Yegor Tamashevich, Kazimir Yanushkevich, Heshmat Noei, Dieter Lott, Andreas Stierle, \\ and Brian Foster
}

\begin{abstract}
Centrifugal barrel polishing (CBP) is a simple and environmentally friendly method that can be applied for mechanical abrasion of the cavity interior in order to remove the mechanically damaged surface after its production. The CBP recipes described in the literature, however, require CBP to be performed in many stages, require long processing times and nevertheless are unable to provide good cavity RF performance without additional chemical processing. Here, we report new results on characterization of cavity surfaces treated with a typical CBP recipe, including the contamination with abrasive particles, plastic deformation and hydrogen contamination, and critically evaluate it. Methods to reduce the depth of significant plastic deformation as well as the modified commercially viable CBP procedure followed by final electropolishing are proposed and tested on samples.
\end{abstract}

Index Terms-Abrasives, linear particle accelerator, materials processing, superconducting materials, Type II superconductors.

\section{$\mathbf{E}$} LECTROPOLISHING (EP) is an important technological step in the current manufacturing process of niobium superconducting radio frequency (SRF) cavities [1]. Firstly, a so-called "bulk" $\mathrm{EP}\left(\mathrm{H}_{2} \mathrm{SO}_{4}\right.$ : $\left.\mathrm{HF}\right)$ is applied to remove a mechanically damaged layer (up to $200 \mu \mathrm{m}$ ) from the interior surface of the cavity left after the rolling and forming steps, followed by annealing $\left(600-900{ }^{\circ} \mathrm{C}\right)$ to degas hydrogen absorbed in this process, a second "light" EP $(10-40 \mu \mathrm{m})$ has to be applied in order to provide a high-quality surface after this annealing in relatively poor vacuum conditions $\left(10^{-5}-10^{-8} \mathrm{mbar}\right)$ [1], [2]. Along with the technological complexity and environmental

Manuscript received September 18, 2017; accepted December 20, 2017. This work was supported by the BMBF Project SRF_RandD. (Corresponding author: Alena Prudnikava.)

A. Prudnikava, A. Stierle, and B. Foster are with the University of Hamburg, Institute of Experimental Physics, Hamburg 22607, Germany, and also with the Deutsches Elektronen Synchrotron, Hamburg 22607, Germany (e-mail: alena.prudnikava@desy.de; andreas.stierle@desy.de; brian.foster@desy.de).

Y. Tamashevich is with the University of Hamburg, Institute of Experimental Physics, Hamburg 22607, Germany. He is now with the HelmholtzZentrum Berlin for Materials and Energy, Berlin 14109, Germany (e-mail: yegor.tamashevich@helmholtz-berlin.de).

K. Yanushkevich is with the Scientific and Practical Materials Research Center, Institute of Semiconductor and Solid State Physics, National Academy of Sciences of Belarus, Minsk 220072, Belarus (e-mail: kazimir@ifttp.bas-net.by).

H. Noei is with the Deutsches Elektronen Synchrotron, Hamburg 22607, Germany (e-mail: Heshmat.noei@desy.de).

D. Lott is with the Institute for Materials Research, Helmholtz-Zentrum Geesthacht, Geesthacht 21502, Germany (e-mail: dieter.lott@hzg.de).

Color versions of one or more of the figures in this paper are available online at http://ieeexplore.ieee.org.

Digital Object Identifier 10.1109/TASC.2018.2791641 unfriendliness, EP has inherently limited efficiency in smoothing out pits at cavity equator areas and inclusions of foreign material.

Centrifugal Barrel Polishing (CBP) is an alternative technique and is free of the aforementioned drawbacks. It has been studied since the 1960s [3]. Considerable contributions to cavity processing by CBP was made by T. Higuchi et al. [4], [5]. During $\mathrm{CBP}$, the cavity fixed in the CBP machine is rapidly rotated with its interior partially filled with abrasives whose characteristic size is reduced at each treatment step, thus gradually improving the surface quality (roughness, amount of plastic deformations). In recent years, a 5-step CBP recipe was investigated by Fermilab and JLab [6], [7], which allows a mirror-smooth cavity surface ( $<15 \mathrm{~nm}$ RMS roughness) to be produced [8], aiming at chemistry-free cavity processing. Despite substantial progress, no reasonable quality factor and accelerating gradient have been achieved without chemical postprocessing [8], [9].

Considering the fact that chemical treatment is the best for achieving the highest surface quality, especially for ductile materials as niobium, our aim is to decrease chemical usage in industrial cavity production by replacing "bulk" EP with CBP which has to be optimized for that purpose.

\section{PREVIOUS Results}

Our activities have been focused on a detailed study of the CBP recipe similar to the one described in [6], [7]. Briefly, a four-step CBP procedure (here steps are numbered as CBP\#1, CBP\#2 and so on), firstly with ceramic triangles (Duramedia ACT), then plastic cones (VF-RG 22), alumina mesh \#600 and finally colloidal silica $(40 \mathrm{~nm})$ as abrasives resulted in a mirror surface finish of single-cell cavity. A technique of surface examination with the use of small removable coupons installed in a "coupon" cavity is described in detail in [10]. In particular, the removal rate from cavity walls as well as SEM investigations of $\mathrm{Nb}$ surface at iris and equator after each CBP step was reported. Drawbacks such as abrasive particles $\left(\mathrm{Al}_{2} \mathrm{O}_{3}\right)$ becoming embedded at CBP\#3, were observed, in agreement with [6]. Moreover, they were found to produce new scratches during the following (final) step (Fig. 1). This problem was partially resolved (i.e., the number of embedded particles was reduced) by additional washing in an ultrasonic bath and renewing the polishing solution at the final CBP step. Field emission studies of a coupon located in the end-tube treated with CBP followed by buffered chemical polishing demonstrated low onset fields of $40 \mathrm{MV} / \mathrm{m}$ (for more detail, see [11]). Additionally, investigation 


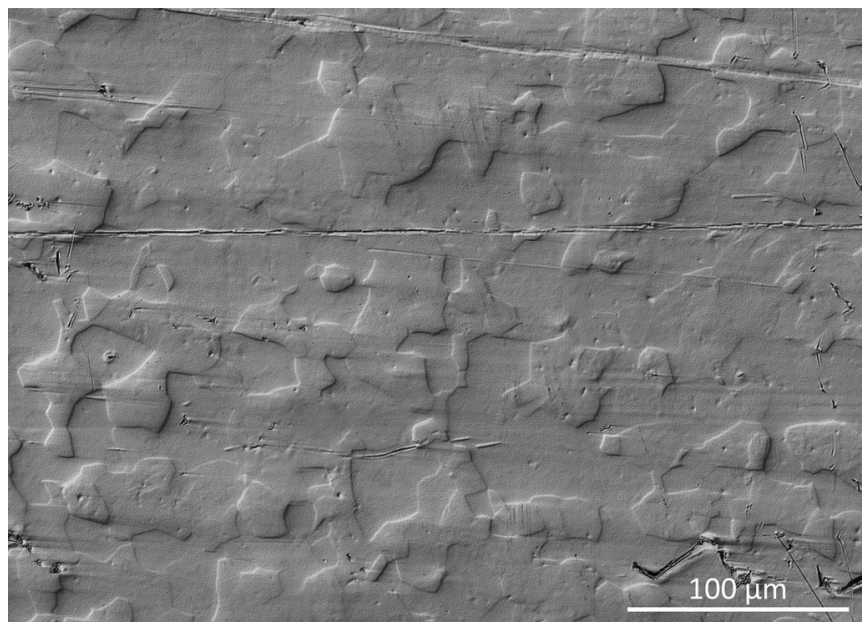

Fig. 1. The surface topography of equator coupon after the full cycle 4step CBP treatment with a mirror surface finish (laser Differential Interference Contrast (DIC) image, 3D laser scanning microscopy). The grain boundaries as well as the freshly produced scratches by the released polishing media which has been used at $\mathrm{CBP \# 3}\left(\mathrm{Al}_{2} \mathrm{O}_{3}\right)$ are visible.

of coupons after each CBP step with laser profilometry showed that alumina mesh made no improvement to the topography and surface roughness [12]. Metallographic preparation of coupons revealed a plastically deformed surface layer especially at equator areas after CBP\#1. On the contrary, the maximal amount of microscale damage after the final CBP treatment was detected by a Vickers hardness test at the end-tube coupons [12]. Precipitation of niobium hydrides upon cooling was observed ex-situ, which evidenced a pronounced hydrogen absorption during CBP, probably enhanced by severe abrasion conditions as demonstrated by the metallographic study.

\section{ONGOING ACTIVITIES}

\section{A. SEM Studies of Nb Coupons After the 4-step CBP}

We have continued to explore the basic 4-step CBP recipe. In particular, surface quality in the different locations of the $1.3 \mathrm{GHz}$ elliptical single-cell cavity having a mirror-smooth surface after the full-cycle CBP with refreshing of the polishing media at final polishing step was further studied by means of coupons and High-Resolution Scanning Electron Microscopy (HR-SEM) with a backscattered electron imaging (BSE) and Energy-dispersive X-ray Spectroscopy (EDX). The use of the backscattered electron imaging, as compared to secondary electron (SE) imaging, due to atomic-number contrast effects allows the inclusions of foreign materials in niobium to be unambiguously distinguished. This is demonstrated in Fig. 2 where SE (a) and a corresponding BSE (b) SEM image of the end-tube coupon with embedded $\mathrm{Al}_{2} \mathrm{O}_{3}$ particles after the basic 4-step CBP treatment are presented. It was revealed that after the final CBP step the surface of the coupons located in the end-tube (the narrowest) area of the cavity contained the highest density of alumina particles, while the coupons installed in the equator area demonstrated mainly pits and scratches produced by these particles (Fig. 2(c)). The estimated dimension of alumina particles in the end-tube area is in the range of $1.5-15 \mu \mathrm{m}$, the
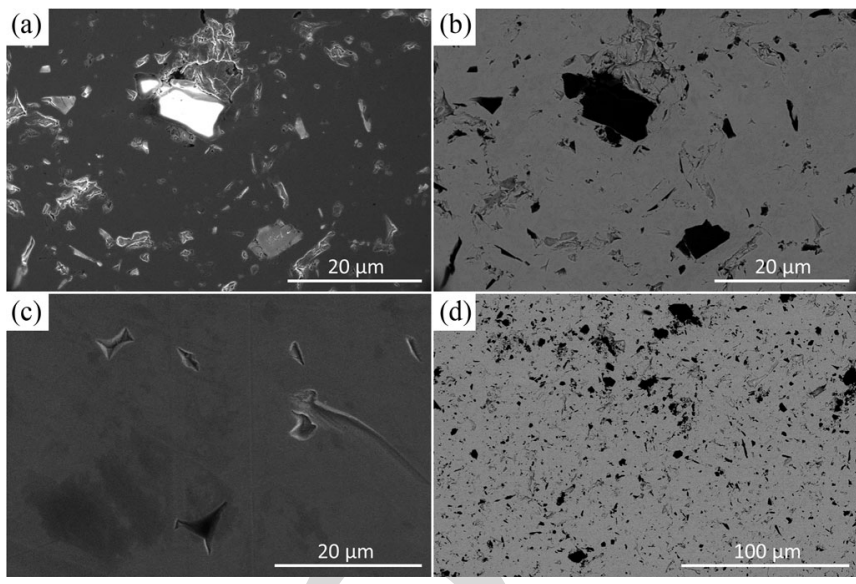

Fig. 2. SE (a) and a corresponding BSE (b) SEM image of the end-tube coupon with embedded $\mathrm{Al}_{2} \mathrm{O}_{3}$ particles after the full cycle 4-step CBP treatment. (c) SE image of the typical defects (pits) found at the equator coupon after the same treatment. (d) BSE image depicting a high degree of particle embedding at end-tube area.
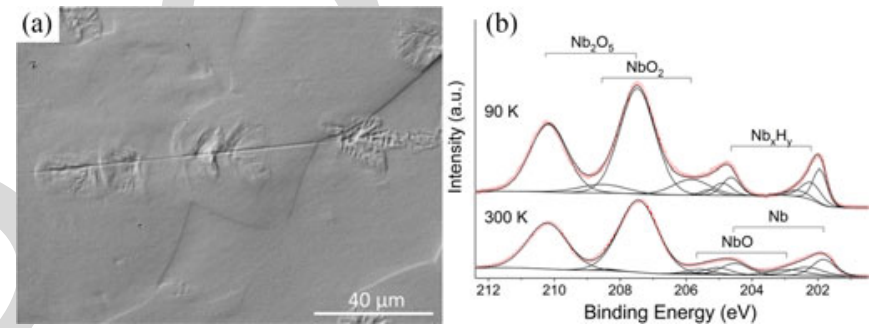

Fig. 3. (a) Laser DIC image depicting the surface relief features left by the precipitated at low temperatures $\mathrm{NbH}_{\mathrm{x}}$. (b) XPS spectra of $\mathrm{Nb} 3 \mathrm{~d}$ region measured at room temperature (lower spectrum) and at $90 \mathrm{~K}$ (upper spectrum).

particle distribution density was 30-40 particles over the area of $25 \mu \mathrm{m} \times 25 \mu \mathrm{m}$. This and previous results demonstrate that alumina mesh \#600 is an inappropriate polishing medium for processing niobium cavities (at least for the current CBP recipe).

In order to remove the observed particles from irises and smooth out the pits at equators, a substantial amount of chemical post processing would be required, which is not in line with our goals.

\section{B. Niobium Hydrides by X-Ray Photoelectron Spectroscopy}

The hydrogen contamination facilitated by mechanical abrasion which is known to be responsible for the $\mathrm{Q}_{0}$ disease phenomenon in CBP-treated cavities [13], was studied by X-ray Photoelectron Spectroscopy (XPS) at room temperature and upon cooling to $90 \mathrm{~K}$ (Fig. 3).

The XPS measurements were carried out using a highresolution 2D delay line detector. A monochromatic $\mathrm{AlK}_{\alpha} \mathrm{X}$-ray source (photon energy $1486.6 \mathrm{eV}$ ) was used as incident radiation [14]. For all spectra, the binding energies were calibrated based on the $\mathrm{C} 1 \mathrm{~s}$ peak at $284.8 \mathrm{~V}$. The area ratio for the $\mathrm{Nb}$ $3 \mathrm{~d}$ spin-orbit doublets was fixed to 1:0.67 with an energy difference of $2.7 \mathrm{eV}$. The existence of Nb-hydride was confirmed with the peaks at low binding energy regions between 201.3 and $203.3 \mathrm{eV}$, which remarkably changed after cooling by shifting to lower binding energies and becoming narrower (Fig. 3(b)). 


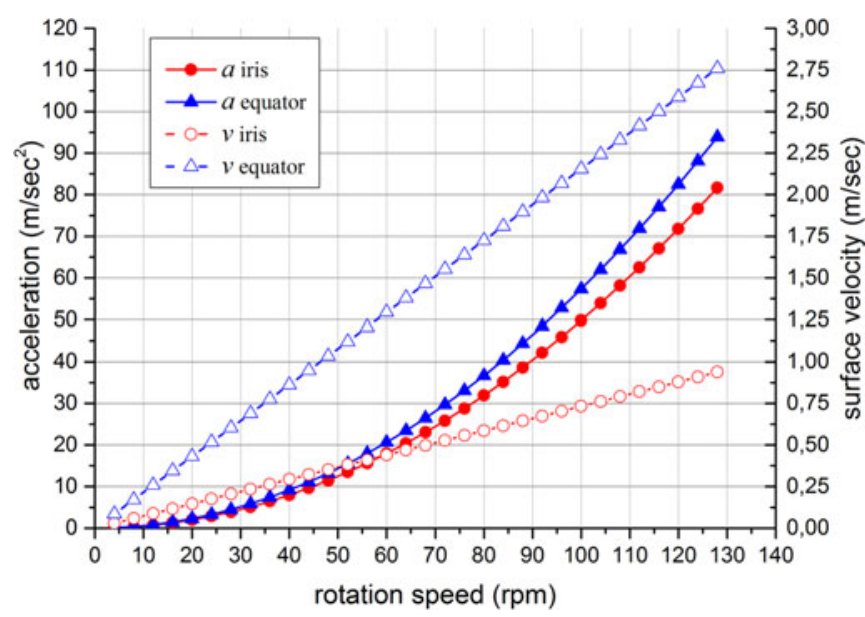

Fig. 4. The calculated centripetal acceleration $a$ (solid lines) and the surfaceto-media velocity $v$ (dashed lines) for different rotation speed of the main shaft.

The way to reduce hydrogen loading during CBP is via the implementation of milder conditions of material removal (abrasion), thus reducing the mechanically deformed layer, or/and decreasing the duration of polishing. Nevertheless, currently at an industrial scale, the problem with the excess amount of hydrogen in cavities is resolved by implementing a so-called high-temperature baking $\left(600-900{ }^{\circ} \mathrm{C}\right)$, which is used to relax the mechanical strain in the cavity and simultaneously degas the absorbed hydrogen. Since this technological step has to be applied anyway, the development of hydrogen-free polishing becomes less important.

\section{Toward Optimization of the Current CBP Recipe}

One way to decrease the plastically deformed layer is to apply a milder polishing condition, i.e., decrease the force pressing the abrasive to the polished cavity surface. The latter is controlled by the mass of polishing media and the rotation speed of the barrels. The amount of abrasive media should provide reasonable polishing efficiency. The barrel rotation speed should not be too low otherwise the polishing media would drop to the lowest part of the cavity after each rotation period. In our CBP machine, the rotation speed of the main drive determines the centrifugal force acting between the polishing media and the cavity walls (see [15]). As the gear ratio of the main drive to the barrel is fixed (1:2), the main shaft rotation speed is the only variable parameter. The centripetal acceleration, $a=\omega^{2} \cdot r$, and the speed of the cavity surface relative to the polishing media, $v=\omega \cdot r$ (where $r$ is the distance from the main shaft to corresponding cavity area), calculated for various main shaft rotation speeds, $\omega$, are shown in Fig. 4.

In order to reduce the depth of damage layer the rotation speed of the main shaft was reduced to $70 \mathrm{rpm}$ instead of 100 rpm during CBP\#1 and CBP\#2. The abrasion rate and the depth of the observed deformed layer are summarized in Table I. The maximal depth of local damage is shown in brackets. The metallographically prepared cross-sections of coupons are shown in [12]. Thus, for CBP\#1 the material abrasion rate decreased by a factor of three while the depth of significant
TABLE I

DAMAGED LAYER THICKNESS AND ABRASION RATE ESTIMATED AFTER CBP\# PERFORMED AT 100 RPM AND 70 RPM ROTATION VELOCITY OF THE BARRELS DURING CBP\#1

\begin{tabular}{lccccc}
\hline \hline Coupon & \multicolumn{2}{c}{ Damaged layer, $\mu \mathrm{m}$} & & \multicolumn{2}{l}{ Abrasion rate, $\mu \mathrm{m} / \mathrm{h}$} \\
\cline { 2 - 3 } \cline { 5 - 6 } & $100 \mathrm{rpm}$ & $70 \mathrm{rpm}$ & & $100 \mathrm{rpm}$ & $70 \mathrm{rpm}$ \\
\hline Tube & $15(25)$ & $10(15)$ & & 3.0 & 0.8 \\
Equator, Cell & $30(70)$ & $15(55)$ & & 9.0 & 3.0 \\
\hline \hline
\end{tabular}

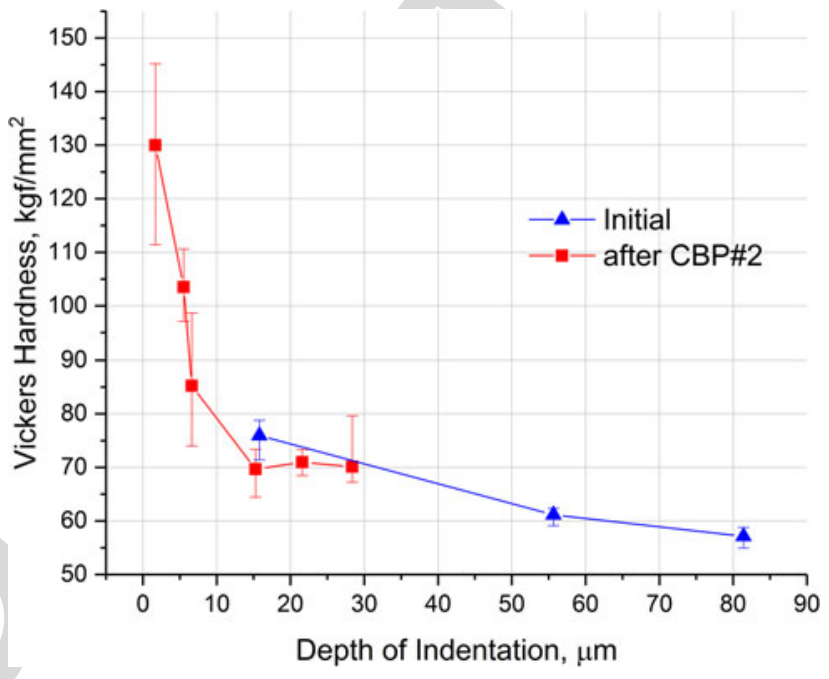

Fig. 5. Vickers hardness measured at the cell area coupon before and after CBP\#2.

deformation in most crucial areas of the cavity decreased by up 172 to $50 \%$ as compared to $100 \mathrm{rpm}$. It is notable, that after CBP\#1 173 followed by CBP\#2 as well as after the standalone CBP\#2, no 174 visible deformations from the standard $100 \mathrm{rpm}$ recipe were 175 observed.

Our earlier SEM studies showed that the CBP\#2 step causes no abrasive embedding into the niobium surface. Therefore, CBP\#2 can be considered for a simplified one-step CBP scheme with chemical post-processing. In this case the duration of the polishing as compared to CBP\#1 should be increased ( $\sim 3$ times) to compensate lower removal rate [10].

The modified scheme was tested using the coupon cavity. Newly prepared coupons were treated with a single CBP\#2 during $20 \mathrm{~h}$ at $100 \mathrm{rpm}$ rotation of the main shaft of CBP machine. $50 \mu \mathrm{m}$ was removed from equator and $16.5 \mu \mathrm{m}$ from irises. The microstructure of the coupons was tested by the Vickers method. Indentations were made with different loads ranging from 0.005 to $0.05 \mathrm{kgf}$ (testers Shimadzu HMV-2000 and Zwick Roell HZV30/ZwickLine). The Vickers hardness values of the equator coupon before and after $\mathrm{CBP} \# 2$ are presented vs the depth of indentation in Fig. 5. The depth of each indentation was measured with a laser microscope (Keyence VK-X100). The presented data are the average of five indentation tests at each load with minimal and maximal values obtained during measurements depicted as error bars. It is seen from the plot that at the indentation depth of $15 \mu \mathrm{m}$, the hardness of the pol- 
TABLE II

Surface Roughness IN $\mu \mathrm{M}$, MEASURED Over AREA OF $270 \mu \mathrm{M} \times 270 \mu \mathrm{M}$ OF CELl COUPON AFTER BASIC CBP, CBP\#2 AND CBP\#2 With EP

\begin{tabular}{lcccc}
\hline \hline Step & $\mathrm{R}_{\mathrm{p}}$ & $\mathrm{R}_{\mathrm{v}}$ & $\mathrm{R}_{\mathrm{z}}$ & $\mathrm{R}_{\mathrm{a}}$ \\
\hline 4-step CBP & $0.74 \pm 0.27$ & $1.54 \pm 0.79$ & $2.28 \pm 0.85$ & $0.11 \pm 0.02$ \\
CBP\#2 & $1.88 \pm 0.41$ & $2.00 \pm 0.24$ & $3.87 \pm 0.65$ & $0.21 \pm 0.01$ \\
CBP\#2+EP & $0.42 \pm 0.08$ & $0.60 \pm 0.13$ & $1.02 \pm 0.21$ & $0.10 \pm 0.01$ \\
\hline
\end{tabular}

$\mathrm{R}_{\mathrm{a}}$ - simple average; $\mathrm{R}_{\mathrm{v}}$ - aver. valley depth; $\mathrm{R}_{\mathrm{z}}$ - aver. peak-to-valley distance; $\mathrm{R}_{\mathrm{p}}-$ aver. peak height.

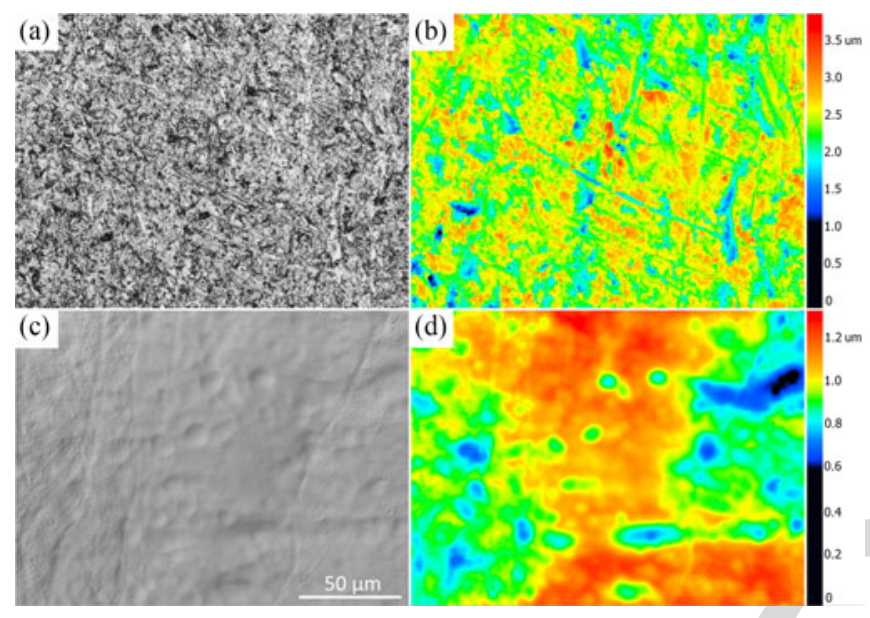

Fig. 6. The surface topography of the cell coupon after CBP\#2 (a, b), and after subsequent EP (c, d). (3D laser scanning microscopy). (a) - laser image; (c) - laser DIC image; (b, d) - height profile images. The scale bar is valid for all the images.

ished material drops to the values of untreated material within the measurement error. This implies that the removal of 15-20 $\mu \mathrm{m}$ material by EP should be sufficient to remove the strain caused by CBP.

It is well established that the initial surface roughness determines the effectiveness of EP to smooth out inhomogeneities. Therefore it is important to verify whether the surface finish provided by CBP\#2 is suitable as a starting condition for EP. We applied $18 \mu \mathrm{m}$ EP to one of the coupons (cell area). Visually, a mirror smooth surface was attained; only in the coupon center was it less perfect. Therefore, the roughness and topography were measured in the central part of the coupon treated with CBP\#2 and following EP where it was less effective (see Table II and Fig. 6). In order to enhance the surface features, DIC processing had to be applied to the laser image of the EP treated surface (Fig. 6(c)). We deem the observed surface inhomogeneities are due to the nature of EP process. Despite this, the roughness improved significantly, i.e., the peak heights were decreased approximately 4.5 times, while valleys were reduced by 3.3 times, resulting in an overall peak-to-valley distance of $1 \mu \mathrm{m}$ over an area of $270 \mu \mathrm{m} \times 270 \mu \mathrm{m}$. Average roughness improved by more than $50 \%$, achieving a value of $0.1 \mu \mathrm{m}$, which is better than for basic 4-step CBP.

$\mathrm{X}$-Ray diffraction (XRD) measurements were made using $\mathrm{CuK}_{\alpha}$ radiation of a standard X-ray tube on a Seifert threecircle diffractometer, equipped with a one-dimensional CCD
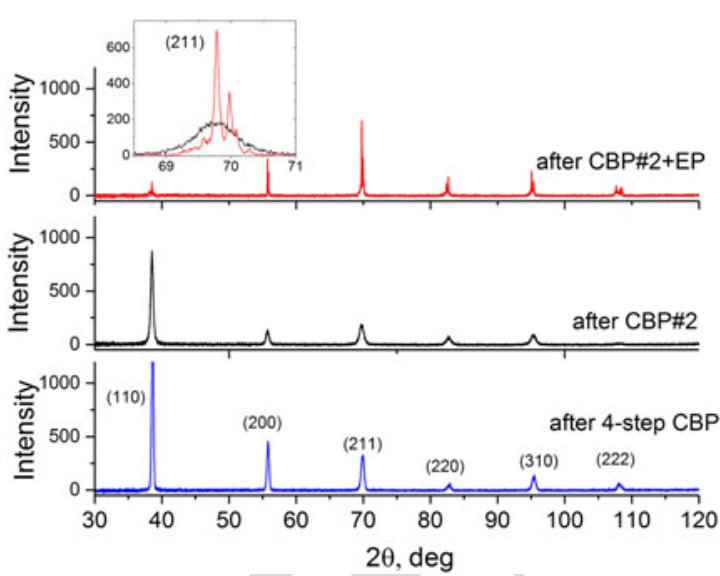

Fig. 7. XRD patterns of $\mathrm{Nb}$ coupons after 4-step CBP, after CBP\#2 and after $\mathrm{CBP} \# 2$ followed by $\mathrm{EP}$ of $18 \mu \mathrm{m}$ (from bottom to up). Insert: magnified view of the Bragg peak centered at $2 \theta=69.76^{\circ}$ of the CBP\#2 coupon before and after EP.

detector. The XRD patterns were measured at a fixed incident 224 angle of $5^{\circ}$ with the scattering angle ranging from $2 \theta=30^{\circ}$ to 225 $120^{\circ}$. The measured XRD patterns (after the background sub- 226 traction) of the cell area coupons treated with basic 4-step CBP, 227 CBP\#2 and following EP, presented in Fig. 7, coincide well with 228 34-0370 and 35-07893 PSPDFWIN data files. The $(h k l)$ indices 229 of Bragg peaks corresponding to bcc $\mathrm{Nb}$ are indicated. While 230 XRD peaks after mechanical processing look alike and rather 231 broad, after additional EP they become so narrow that the $\mathrm{CuK}_{\alpha} \quad 232$ fine structure of the X-ray source become visible in the diffrac- 233 tion pattern (Fig. 7, insert). These peaks are to be further ana- 234 lyzed to determine whether additional phases were formed upon 235 EP (oxides, etc.). The mean crystallite size determined using 236 the Scherrer equation [16] for the CBP\#2 treated coupon was 237 $12.98 \mathrm{~nm}$, while after EP the peak width reaches the resolution 238 limit of the experimental setup. Therefore, only a lower limit 239 of the mean crystallite size of $133.4 \mathrm{~nm}$ could be determined, 240 with the corresponding density of dislocations, i.e. the length 241 of dislocation lines per unit volume of the crystal, 5.9 $10^{-17} 242$ and $5.6 \cdot 10^{-19} \mathrm{~cm}^{-2}$ [17]. The similarly obtained values for the 243 coupon processed with basic 4-step CBP are just $25.22 \mathrm{~nm}$ and 244 $1.57 \cdot 10^{-17} \mathrm{~cm}^{-2}$ despite the mirror-smooth surface.

\section{CONCLUSION}

In this paper, we performed characterization of niobium sam- 247 ples processed with CBP using a coupon cavity. We updated 248 the results of our recent studies of the basic 4-step CBP recipe. 249 Particularly, with backscattered electron imaging, the embed- 250 ded alumina particles were precisely visualized at the narrowest 251 area of the cavity, while at equator/cell area coupons mainly pits 252 left by these particles were present. Summing up our findings, 253 we conclude that alumina mesh \#600 is an inappropriate pol- 254 ishing medium in the current CBP recipe. For the first time, a 255 large plastically deformed layer produced in a cavity interior sur- 256 face during CBP was demonstrated using metallographic sample 257 preparation. The effect of the rotation speed of the barrel was 258 demonstrated in practice. A simplified scheme for cavity prepa- 259 ration involving both CBP and EP is proposed which is likely 260 
to be competitive with the present industrial cavity preparation procedure.

The authors would like to thank X. Singer and W. Singer for providing the high quality niobium for the current investigation. We are very grateful to our colleagues A. Navitski, A. Ermakov, J. Schaffran for the enormous input for the startup of the current studies. We express our thanks to KEK colleagues and especially Takayuki Saeki and Shigeki Kato for the fabrication of the coupon cavity.

[1] L. Lilje et al., "Improved surface treatment of the superconducting TESLA cavities," Nucl. Instrum. Methods Phys. Res. A, Accel., Spectrom., Detect. Assoc. Equip., vol. 516, no. 2, pp. 213-227, 2004.

[2] Z. Sung, A. Dzyuba, P. Lee, D. Larbalestier, and L. Cooley, "Evidence of incomplete annealing at $800^{\circ} \mathrm{C}$ and the effects of $120^{\circ} \mathrm{C}$ baking on the crystal orientation and the surface superconducting properties of coldworked and chemically polished Nb," Supercond. Sci. Technol., vol. 28, no. 7, 2015, Art. no. 075003 .

[3] H. Kobayashi and M. Matsunaga, "Some experiments on centrifugal barrel finishing," Met Finish, vol. 64, no. 5, pp. 57-60, 1966.

[4] T. Higuchi et al., "Investigation on barrel polishing for superconducting niobium cavities," Proc. 1995 Workshop RF Supercond., Gif-sur-Yvette, France, 1995, pp. 723-727.

[5] T. Higuchi and K. Saito, "Development of hydrogen-free EP and hydrogen absorption phenomena," in Proc. 11th Workshop RF Supercond., Lübek, Germany, 2003, pp. 572-578.

[6] A. D. Palczewski, H. Tian, O. Trofimova, and C. E. Reece, "Detailed surface analysis of incremental centrifugal barrel polishing (CBP) of singlecrystal niobium samples," in Proc. Int. Conf. RF Supercond., Chicago, IL, USA, 2011, pp. 908-911.
[7] A. Palczewski, C. Cooper, B. Bullock, S. Joshi, A. Rossi, and A. Navitski, "R\&D progress in SRF surface preparation with centrifugal barrel polishing (CBP) for both $\mathrm{Nb}$ and $\mathrm{Cu}$," in Proc. Int. Conf. RF Supercond., Paris, France, 2013, pp. 395-400.

[8] C. Cooper and L. Cooley, "Mirror-smooth surfaces and repair of defects in superconducting RF cavities by mechanical polishing," Supercond. Sci. Technol., vol. 26, no. 1, 2012, Art. no. 015011.

[9] A. Palczewski, R. Geng, and H. Tian, "Optimizing Centrifugal barrel polishing for mirror finish SRF cavity and RF tests at Jefferson Lab," in Proc. Int. Particle Accel. Conf., New Orleans, LA, USA, 2012, pp. 24352437.

[10] A. Navitski, E. Elsen, B. Foster, A Prudnikava, and Y. Tamashevich, 303 "Surface analyses and optimization of centrifugal barrel polishing of $\mathrm{Nb} 304$ cavities," in Proc. Int. Conf. RF Supercond., Whistler, BC, Canada, 2015, 305 pp. 286-290.

[11] S. Lagotzky, G. Müller, A. Navitski, A. Prudnikava, and Y. Tamashevich, 307 "Field emission investigation of centrifugal-barrel-polished NB samples," 308 in Proc. Int. Conf. RF Supercond., Whistler, BC, Canada, 2015, pp. 237- 309 241.

[12] A. Prudnikava, B. Foster, Y. Tamashevich, and A. Ermakov, "R\&D 311 Activities on centrifugal barrel polishing of $1.3 \mathrm{GHz}$ niobium cavities 312 at DESY/University of hamburg," in Proc. Int. Conf. RF Supercond., 313 Lanzhou, China, 2017, Art. no. TUPB111, 4 pages. (in press).

[13] B. Visentin, J. Charrier, B. Coadou, and D. Roudier, "Cavity baking: A 315 cure for the high accelerator field $\mathrm{Q}_{0}$ drop," in Proc. 1999 Workshop RF 316 Supercond., 1999, pp. 198-202.

[14] A. Stierle, T. F. Keller, H. Noei, V. Vonk, and R. Roehlsberger, "DESY 318 NanoLab," J. Large-Scale Res. Facilities JLSRF, vol. 2, no. A76, 2016, 319 pp. 1-6. 320

[15] Y. Tamashevich "Diagnostics and treatment of $1.3 \mathrm{GHz} \mathrm{Nb}$ cavities," 321 Ph.D. dissertation, Dept. Physics, Univ. Hamburg, Hamburg, Germany, 322 2016.

[16] P. Scherrer "Bestimmung der inneren Struktur und der Größe von kol- 324 loidteilchen mittels röntgenstrahlen," Kolloidchemie Ein Lehrbuch, Berlin, 325 Germany: Springer-Verlag, 1912, pp. 387-409 .

[17] G. Williamson, and R. Smallman, "III. Dislocation densities in some 327 annealed and cold-worked metals from measurements on the X-ray debye- 328 scherrer spectrum," Philosoph. Mag., vol. 1, no. 1, pp. 34-46, 1956. 


\title{
Toward Optimization of Centrifugal Barrel Polishing Procedure for Treatment of Niobium Cavities
}

\author{
Alena Prudnikava, Yegor Tamashevich, Kazimir Yanushkevich, Heshmat Noei, Dieter Lott, Andreas Stierle, \\ and Brian Foster
}

\begin{abstract}
Centrifugal barrel polishing (CBP) is a simple and environmentally friendly method that can be applied for mechanical abrasion of the cavity interior in order to remove the mechanically damaged surface after its production. The CBP recipes described in the literature, however, require CBP to be performed in many stages, require long processing times and nevertheless are unable to provide good cavity RF performance without additional chemical processing. Here, we report new results on characterization of cavity surfaces treated with a typical CBP recipe, including the contamination with abrasive particles, plastic deformation and hydrogen contamination, and critically evaluate it. Methods to reduce the depth of significant plastic deformation as well as the modified commercially viable CBP procedure followed by final electropolishing are proposed and tested on samples.
\end{abstract}

Index Terms-Abrasives, linear particle accelerator, materials processing, superconducting materials, Type II superconductors.

\section{$\mathbf{E}$} LECTROPOLISHING (EP) is an important technological step in the current manufacturing process of niobium superconducting radio frequency (SRF) cavities [1]. Firstly, a so-called "bulk" $\mathrm{EP}\left(\mathrm{H}_{2} \mathrm{SO}_{4}\right.$ : $\left.\mathrm{HF}\right)$ is applied to remove a mechanically damaged layer (up to $200 \mu \mathrm{m}$ ) from the interior surface of the cavity left after the rolling and forming steps, followed by annealing $\left(600-900{ }^{\circ} \mathrm{C}\right)$ to degas hydrogen absorbed in this process, a second "light" EP $(10-40 \mu \mathrm{m})$ has to be applied in order to provide a high-quality surface after this annealing in relatively poor vacuum conditions $\left(10^{-5}-10^{-8} \mathrm{mbar}\right)$ [1], [2]. Along with the technological complexity and environmental

Manuscript received September 18, 2017; accepted December 20, 2017. This work was supported by the BMBF Project SRF_RandD. (Corresponding author: Alena Prudnikava.)

A. Prudnikava, A. Stierle, and B. Foster are with the University of Hamburg, Institute of Experimental Physics, Hamburg 22607, Germany, and also with the Deutsches Elektronen Synchrotron, Hamburg 22607, Germany (e-mail: alena.prudnikava@desy.de; andreas.stierle@desy.de; brian.foster@desy.de).

Y. Tamashevich is with the University of Hamburg, Institute of Experimental Physics, Hamburg 22607, Germany. He is now with the HelmholtzZentrum Berlin for Materials and Energy, Berlin 14109, Germany (e-mail: yegor.tamashevich@helmholtz-berlin.de).

K. Yanushkevich is with the Scientific and Practical Materials Research Center, Institute of Semiconductor and Solid State Physics, National Academy of Sciences of Belarus, Minsk 220072, Belarus (e-mail: kazimir@ifttp.bas-net.by).

H. Noei is with the Deutsches Elektronen Synchrotron, Hamburg 22607, Germany (e-mail: Heshmat.noei@desy.de).

D. Lott is with the Institute for Materials Research, Helmholtz-Zentrum Geesthacht, Geesthacht 21502, Germany (e-mail: dieter.lott@hzg.de).

Color versions of one or more of the figures in this paper are available online at http://ieeexplore.ieee.org.

Digital Object Identifier 10.1109/TASC.2018.2791641 unfriendliness, EP has inherently limited efficiency in smoothing out pits at cavity equator areas and inclusions of foreign material.

Centrifugal Barrel Polishing (CBP) is an alternative technique and is free of the aforementioned drawbacks. It has been studied since the 1960s [3]. Considerable contributions to cavity processing by CBP was made by T. Higuchi et al. [4], [5]. During $\mathrm{CBP}$, the cavity fixed in the CBP machine is rapidly rotated with its interior partially filled with abrasives whose characteristic size is reduced at each treatment step, thus gradually improving the surface quality (roughness, amount of plastic deformations). In recent years, a 5-step CBP recipe was investigated by Fermilab and JLab [6], [7], which allows a mirror-smooth cavity surface ( $<15 \mathrm{~nm}$ RMS roughness) to be produced [8], aiming at chemistry-free cavity processing. Despite substantial progress, no reasonable quality factor and accelerating gradient have been achieved without chemical postprocessing [8], [9].

Considering the fact that chemical treatment is the best for achieving the highest surface quality, especially for ductile materials as niobium, our aim is to decrease chemical usage in industrial cavity production by replacing "bulk" EP with CBP which has to be optimized for that purpose.

\section{PRevious Results}

Our activities have been focused on a detailed study of the CBP recipe similar to the one described in [6], [7]. Briefly, a four-step CBP procedure (here steps are numbered as CBP\#1, CBP\#2 and so on), firstly with ceramic triangles (Duramedia ACT), then plastic cones (VF-RG 22), alumina mesh \#600 and finally colloidal silica $(40 \mathrm{~nm})$ as abrasives resulted in a mirror surface finish of single-cell cavity. A technique of surface examination with the use of small removable coupons installed in a "coupon" cavity is described in detail in [10]. In particular, the removal rate from cavity walls as well as SEM investigations of $\mathrm{Nb}$ surface at iris and equator after each CBP step was reported. Drawbacks such as abrasive particles $\left(\mathrm{Al}_{2} \mathrm{O}_{3}\right)$ becom-

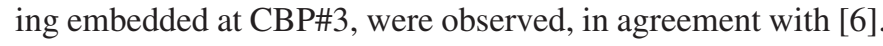
Moreover, they were found to produce new scratches during the following (final) step (Fig. 1). This problem was partially resolved (i.e., the number of embedded particles was reduced) by additional washing in an ultrasonic bath and renewing the polishing solution at the final CBP step. Field emission studies of a coupon located in the end-tube treated with CBP followed by buffered chemical polishing demonstrated low onset fields of $40 \mathrm{MV} / \mathrm{m}$ (for more detail, see [11]). Additionally, investigation 


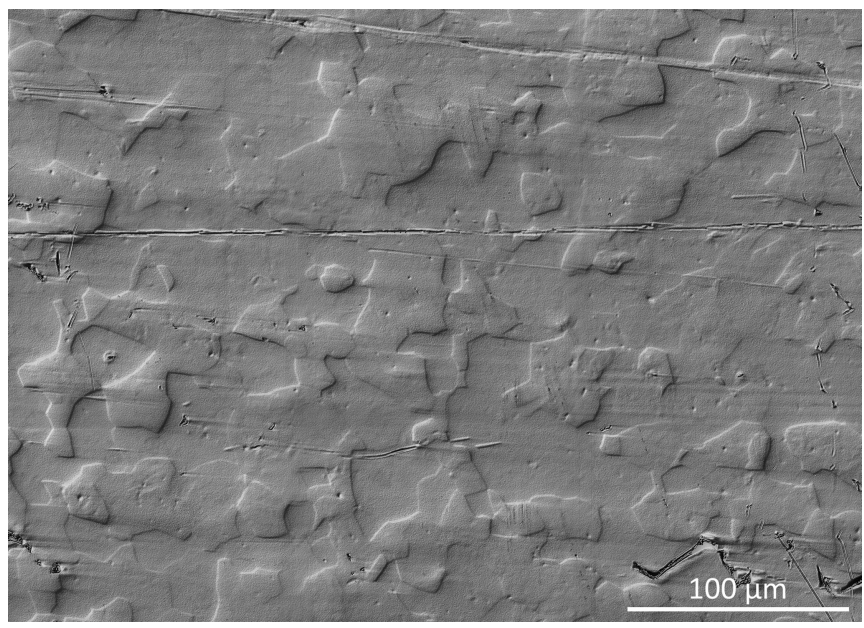

Fig. 1. The surface topography of equator coupon after the full cycle 4step CBP treatment with a mirror surface finish (laser Differential Interference Contrast (DIC) image, 3D laser scanning microscopy). The grain boundaries as well as the freshly produced scratches by the released polishing media which has been used at $\mathrm{CBP \# 3}\left(\mathrm{Al}_{2} \mathrm{O}_{3}\right)$ are visible.

of coupons after each CBP step with laser profilometry showed that alumina mesh made no improvement to the topography and surface roughness [12]. Metallographic preparation of coupons revealed a plastically deformed surface layer especially at equator areas after CBP\#1. On the contrary, the maximal amount of microscale damage after the final CBP treatment was detected by a Vickers hardness test at the end-tube coupons [12]. Precipitation of niobium hydrides upon cooling was observed ex-situ, which evidenced a pronounced hydrogen absorption during CBP, probably enhanced by severe abrasion conditions as demonstrated by the metallographic study.

\section{ONGOING ACTIVITIES}

\section{A. SEM Studies of Nb Coupons After the 4-step CBP}

We have continued to explore the basic 4-step CBP recipe. In particular, surface quality in the different locations of the $1.3 \mathrm{GHz}$ elliptical single-cell cavity having a mirror-smooth surface after the full-cycle CBP with refreshing of the polishing media at final polishing step was further studied by means of coupons and High-Resolution Scanning Electron Microscopy (HR-SEM) with a backscattered electron imaging (BSE) and Energy-dispersive X-ray Spectroscopy (EDX). The use of the backscattered electron imaging, as compared to secondary electron (SE) imaging, due to atomic-number contrast effects allows the inclusions of foreign materials in niobium to be unambiguously distinguished. This is demonstrated in Fig. 2 where SE (a) and a corresponding BSE (b) SEM image of the end-tube coupon with embedded $\mathrm{Al}_{2} \mathrm{O}_{3}$ particles after the basic 4-step CBP treatment are presented. It was revealed that after the final CBP step the surface of the coupons located in the end-tube (the narrowest) area of the cavity contained the highest density of alumina particles, while the coupons installed in the equator area demonstrated mainly pits and scratches produced by these particles (Fig. 2(c)). The estimated dimension of alumina particles in the end-tube area is in the range of $1.5-15 \mu \mathrm{m}$, the
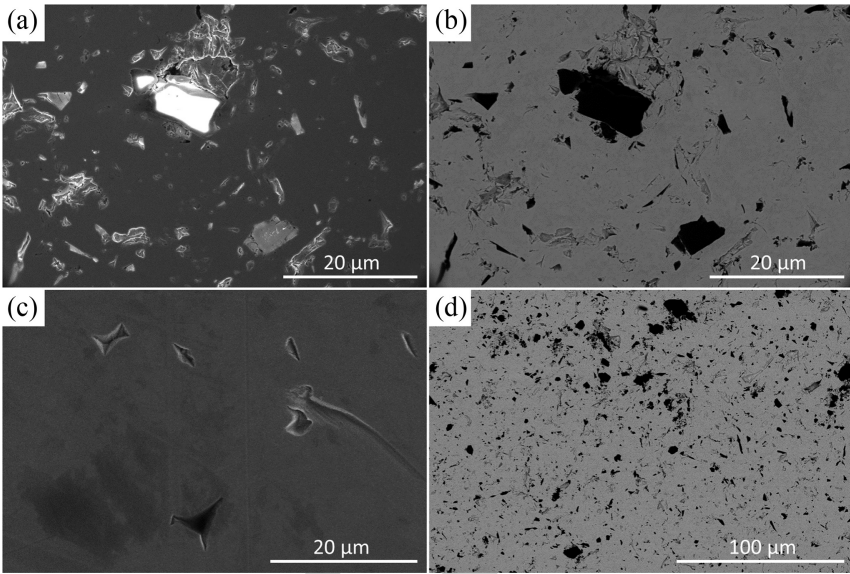

Fig. 2. SE (a) and a corresponding BSE (b) SEM image of the end-tube coupon with embedded $\mathrm{Al}_{2} \mathrm{O}_{3}$ particles after the full cycle 4-step CBP treatment. (c) SE image of the typical defects (pits) found at the equator coupon after the same treatment. (d) BSE image depicting a high degree of particle embedding at end-tube area.
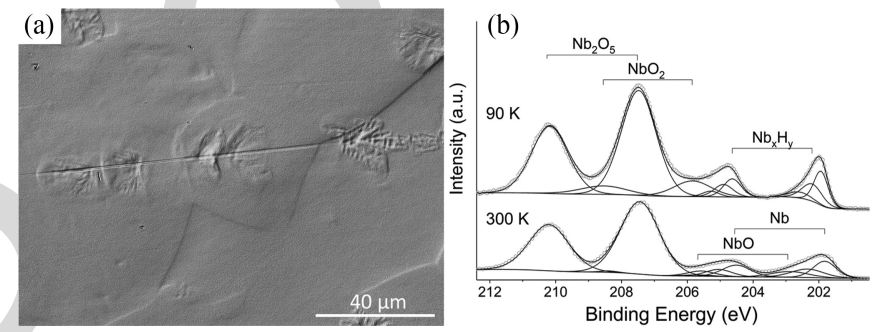

Fig. 3. (a) Laser DIC image depicting the surface relief features left by the precipitated at low temperatures $\mathrm{NbH}_{\mathrm{x}}$. (b) XPS spectra of $\mathrm{Nb} 3 \mathrm{~d}$ region measured at room temperature (lower spectrum) and at $90 \mathrm{~K}$ (upper spectrum).

particle distribution density was 30-40 particles over the area of $25 \mu \mathrm{m} \times 25 \mu \mathrm{m}$. This and previous results demonstrate that alumina mesh \#600 is an inappropriate polishing medium for processing niobium cavities (at least for the current CBP recipe).

In order to remove the observed particles from irises and smooth out the pits at equators, a substantial amount of chemical post processing would be required, which is not in line with our goals.

\section{B. Niobium Hydrides by X-Ray Photoelectron Spectroscopy}

The hydrogen contamination facilitated by mechanical abrasion which is known to be responsible for the $\mathrm{Q}_{0}$ disease phenomenon in CBP-treated cavities [13], was studied by X-ray Photoelectron Spectroscopy (XPS) at room temperature and upon cooling to $90 \mathrm{~K}$ (Fig. 3).

The XPS measurements were carried out using a highresolution 2D delay line detector. A monochromatic $\mathrm{AlK}_{\alpha} \mathrm{X}$-ray source (photon energy $1486.6 \mathrm{eV}$ ) was used as incident radiation [14]. For all spectra, the binding energies were calibrated based on the $\mathrm{C} 1 \mathrm{~s}$ peak at $284.8 \mathrm{~V}$. The area ratio for the $\mathrm{Nb}$ $3 \mathrm{~d}$ spin-orbit doublets was fixed to 1:0.67 with an energy difference of $2.7 \mathrm{eV}$. The existence of Nb-hydride was confirmed with the peaks at low binding energy regions between 201.3 and $203.3 \mathrm{eV}$, which remarkably changed after cooling by shifting to lower binding energies and becoming narrower (Fig. 3(b)). 


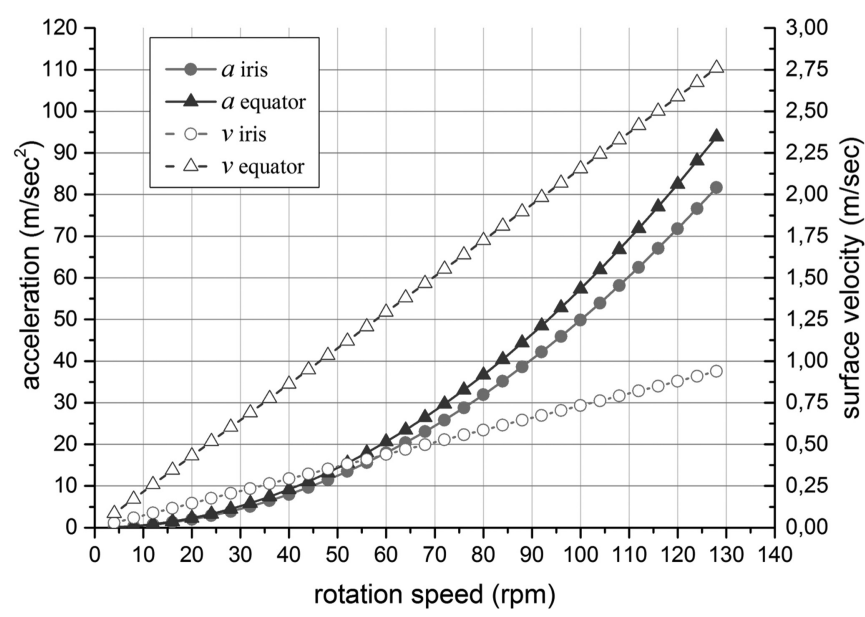

Fig. 4. The calculated centripetal acceleration $a$ (solid lines) and the surfaceto-media velocity $v$ (dashed lines) for different rotation speed of the main shaft.

The way to reduce hydrogen loading during CBP is via the implementation of milder conditions of material removal (abrasion), thus reducing the mechanically deformed layer, or/and decreasing the duration of polishing. Nevertheless, currently at an industrial scale, the problem with the excess amount of hydrogen in cavities is resolved by implementing a so-called high-temperature baking $\left(600-900{ }^{\circ} \mathrm{C}\right)$, which is used to relax the mechanical strain in the cavity and simultaneously degas the absorbed hydrogen. Since this technological step has to be applied anyway, the development of hydrogen-free polishing becomes less important.

\section{Toward Optimization of the Current CBP Recipe}

One way to decrease the plastically deformed layer is to apply a milder polishing condition, i.e., decrease the force pressing the abrasive to the polished cavity surface. The latter is controlled by the mass of polishing media and the rotation speed of the barrels. The amount of abrasive media should provide reasonable polishing efficiency. The barrel rotation speed should not be too low otherwise the polishing media would drop to the lowest part of the cavity after each rotation period. In our CBP machine, the rotation speed of the main drive determines the centrifugal force acting between the polishing media and the cavity walls (see [15]). As the gear ratio of the main drive to the barrel is fixed (1:2), the main shaft rotation speed is the only variable parameter. The centripetal acceleration, $a=\omega^{2} \cdot r$, and the speed of the cavity surface relative to the polishing media, $v=\omega \cdot r$ (where $r$ is the distance from the main shaft to corresponding cavity area), calculated for various main shaft rotation speeds, $\omega$, are shown in Fig. 4.

In order to reduce the depth of damage layer the rotation speed of the main shaft was reduced to $70 \mathrm{rpm}$ instead of 100 rpm during CBP\#1 and CBP\#2. The abrasion rate and the depth of the observed deformed layer are summarized in Table I. The maximal depth of local damage is shown in brackets. The metallographically prepared cross-sections of coupons are shown in [12]. Thus, for CBP\#1 the material abrasion rate decreased by a factor of three while the depth of significant
TABLE I

DAMAGED LAYER THICKNESS AND ABRASION RATE ESTIMATED AFTER CBP\# PERFORMED AT 100 RPM AND 70 RPM ROTATION VELOCITY OF THE BARRELS DURING CBP\#1

\begin{tabular}{lccccc}
\hline \hline Coupon & \multicolumn{2}{c}{ Damaged layer, $\mu \mathrm{m}$} & & \multicolumn{2}{l}{ Abrasion rate, $\mu \mathrm{m} / \mathrm{h}$} \\
\cline { 2 - 3 } \cline { 5 - 6 } & $100 \mathrm{rpm}$ & $70 \mathrm{rpm}$ & & $100 \mathrm{rpm}$ & $70 \mathrm{rpm}$ \\
\hline Tube & $15(25)$ & $10(15)$ & & 3.0 & 0.8 \\
Equator, Cell & $30(70)$ & $15(55)$ & & 9.0 & 3.0 \\
\hline \hline
\end{tabular}

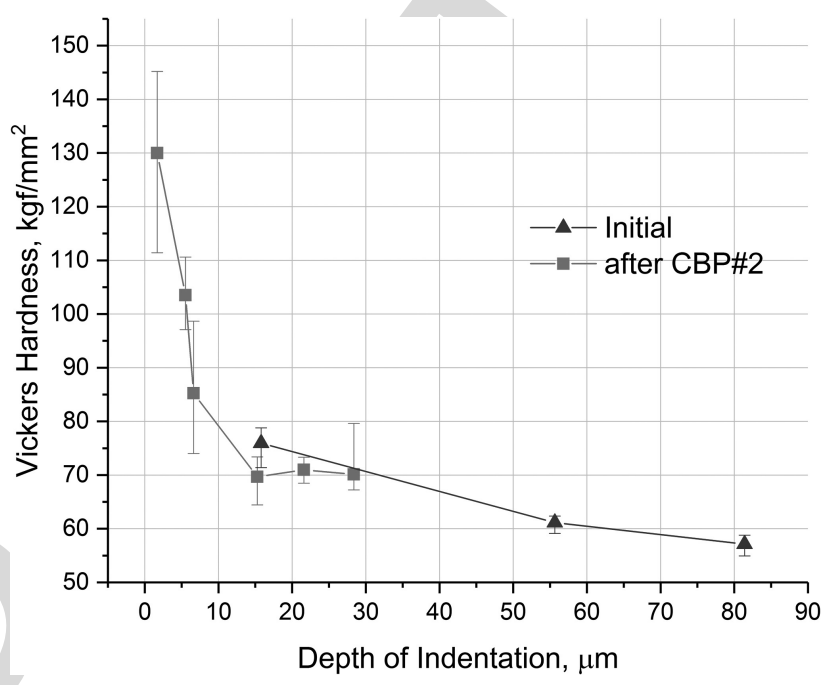

Fig. 5. Vickers hardness measured at the cell area coupon before and after CBP\#2.

deformation in most crucial areas of the cavity decreased by up to $50 \%$ as compared to $100 \mathrm{rpm}$. It is notable, that after CBP\#1 followed by CBP\#2 as well as after the standalone CBP\#2, no visible deformations from the standard $100 \mathrm{rpm}$ recipe were observed.

Our earlier SEM studies showed that the CBP\#2 step causes no abrasive embedding into the niobium surface. Therefore, CBP\#2 can be considered for a simplified one-step CBP scheme with chemical post-processing. In this case the duration of the polishing as compared to CBP\#1 should be increased ( $\sim 3$ times) to compensate lower removal rate [10].

The modified scheme was tested using the coupon cavity. Newly prepared coupons were treated with a single CBP\#2 during $20 \mathrm{~h}$ at $100 \mathrm{rpm}$ rotation of the main shaft of CBP machine. $50 \mu \mathrm{m}$ was removed from equator and $16.5 \mu \mathrm{m}$ from irises. The microstructure of the coupons was tested by the Vickers method. Indentations were made with different loads ranging from 0.005 to $0.05 \mathrm{kgf}$ (testers Shimadzu HMV-2000 and Zwick Roell HZV30/ZwickLine). The Vickers hardness values of the equator coupon before and after $\mathrm{CBP} \# 2$ are presented vs the depth of indentation in Fig. 5. The depth of each indentation was measured with a laser microscope (Keyence VK-X100). The presented data are the average of five indentation tests at each load with minimal and maximal values obtained during measurements depicted as error bars. It is seen from the plot that at the indentation depth of $15 \mu \mathrm{m}$, the hardness of the pol- 
TABLE II

Surface Roughness IN $\mu \mathrm{M}$, MEASURED Over AREA OF $270 \mu \mathrm{M} \times 270 \mu \mathrm{M}$ of Cell COUPON AFTER BAsic CBP, CBP\#2 AND CBP\#2 With EP

\begin{tabular}{lcccc}
\hline \hline Step & $\mathrm{R}_{\mathrm{p}}$ & $\mathrm{R}_{\mathrm{v}}$ & $\mathrm{R}_{\mathrm{z}}$ & $\mathrm{R}_{\mathrm{a}}$ \\
\hline 4-step CBP & $0.74 \pm 0.27$ & $1.54 \pm 0.79$ & $2.28 \pm 0.85$ & $0.11 \pm 0.02$ \\
CBP\#2 & $1.88 \pm 0.41$ & $2.00 \pm 0.24$ & $3.87 \pm 0.65$ & $0.21 \pm 0.01$ \\
CBP\#2+EP & $0.42 \pm 0.08$ & $0.60 \pm 0.13$ & $1.02 \pm 0.21$ & $0.10 \pm 0.01$ \\
\hline \hline
\end{tabular}

$\mathrm{R}_{\mathrm{a}}$ - simple average; $\mathrm{R}_{\mathrm{v}}$ - aver. valley depth; $\mathrm{R}_{\mathrm{z}}$ - aver. peak-to-valley distance; $\mathrm{R}_{\mathrm{p}}-$ aver. peak height.

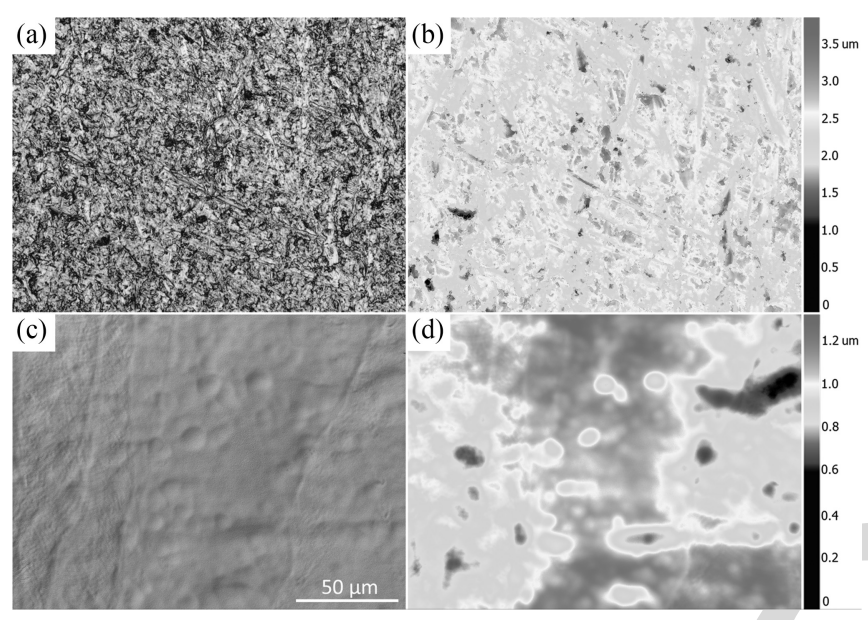

Fig. 6. The surface topography of the cell coupon after $\operatorname{CBP\# 2~(a,b),~and~}$ after subsequent EP (c, d). (3D laser scanning microscopy). (a) - laser image; (c) - laser DIC image; (b, d) - height profile images. The scale bar is valid for all the images.

ished material drops to the values of untreated material within the measurement error. This implies that the removal of 15-20 $\mu \mathrm{m}$ material by EP should be sufficient to remove the strain caused by CBP.

It is well established that the initial surface roughness determines the effectiveness of EP to smooth out inhomogeneities. Therefore it is important to verify whether the surface finish provided by CBP\#2 is suitable as a starting condition for EP. We applied $18 \mu \mathrm{m}$ EP to one of the coupons (cell area). Visually, a mirror smooth surface was attained; only in the coupon center was it less perfect. Therefore, the roughness and topography were measured in the central part of the coupon treated with CBP\#2 and following EP where it was less effective (see Table II and Fig. 6). In order to enhance the surface features, DIC processing had to be applied to the laser image of the EP treated surface (Fig. 6(c)). We deem the observed surface inhomogeneities are due to the nature of EP process. Despite this, the roughness improved significantly, i.e., the peak heights were decreased approximately 4.5 times, while valleys were reduced by 3.3 times, resulting in an overall peak-to-valley distance of $1 \mu \mathrm{m}$ over an area of $270 \mu \mathrm{m} \times 270 \mu \mathrm{m}$. Average roughness improved by more than $50 \%$, achieving a value of $0.1 \mu \mathrm{m}$, which is better than for basic 4-step CBP.

$\mathrm{X}$-Ray diffraction (XRD) measurements were made using $\mathrm{CuK}_{\alpha}$ radiation of a standard X-ray tube on a Seifert threecircle diffractometer, equipped with a one-dimensional CCD
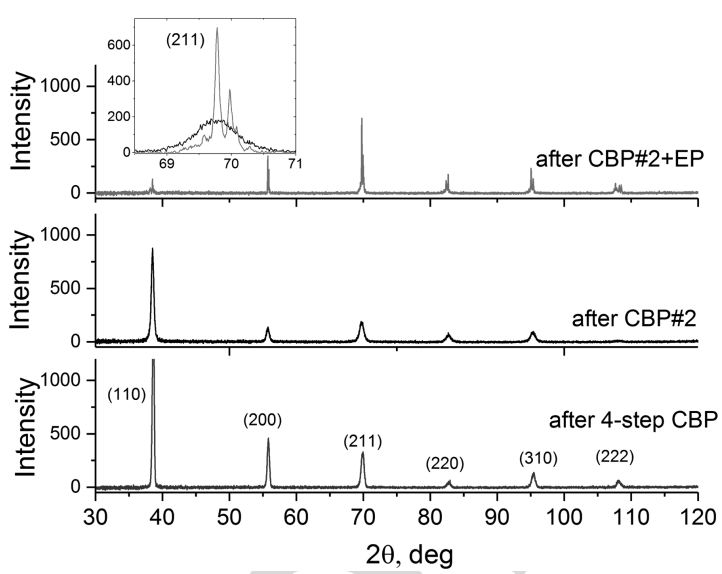

Fig. 7. XRD patterns of $\mathrm{Nb}$ coupons after 4-step CBP, after CBP\#2 and after $\mathrm{CBP} \# 2$ followed by $\mathrm{EP}$ of $18 \mu \mathrm{m}$ (from bottom to up). Insert: magnified view of the Bragg peak centered at $2 \theta=69.76^{\circ}$ of the CBP\#2 coupon before and after EP.

detector. The XRD patterns were measured at a fixed incident 224 angle of $5^{\circ}$ with the scattering angle ranging from $2 \theta=30^{\circ}$ to 225 $120^{\circ}$. The measured XRD patterns (after the background sub- 226 traction) of the cell area coupons treated with basic 4-step CBP, 227 CBP\#2 and following EP, presented in Fig. 7, coincide well with 228 34-0370 and 35-07893 PSPDFWIN data files. The $(h k l)$ indices 229 of Bragg peaks corresponding to bcc $\mathrm{Nb}$ are indicated. While 230 XRD peaks after mechanical processing look alike and rather 231 broad, after additional EP they become so narrow that the $\mathrm{CuK}_{\alpha} \quad 232$ fine structure of the X-ray source become visible in the diffrac- 233 tion pattern (Fig. 7, insert). These peaks are to be further ana- 234 lyzed to determine whether additional phases were formed upon 235 EP (oxides, etc.). The mean crystallite size determined using 236 the Scherrer equation [16] for the CBP\#2 treated coupon was 237 $12.98 \mathrm{~nm}$, while after EP the peak width reaches the resolution 238 limit of the experimental setup. Therefore, only a lower limit 239 of the mean crystallite size of $133.4 \mathrm{~nm}$ could be determined, 240 with the corresponding density of dislocations, i.e. the length 241 of dislocation lines per unit volume of the crystal, 5.9 $10^{-17} 242$ and $5.6 \cdot 10^{-19} \mathrm{~cm}^{-2}$ [17]. The similarly obtained values for the 243 coupon processed with basic 4-step CBP are just $25.22 \mathrm{~nm}$ and 244 $1.57 \cdot 10^{-17} \mathrm{~cm}^{-2}$ despite the mirror-smooth surface.

\section{CONCLUSION}

In this paper, we performed characterization of niobium sam- 247 ples processed with CBP using a coupon cavity. We updated 248 the results of our recent studies of the basic 4-step CBP recipe. 249 Particularly, with backscattered electron imaging, the embed- 250 ded alumina particles were precisely visualized at the narrowest 251 area of the cavity, while at equator/cell area coupons mainly pits 252 left by these particles were present. Summing up our findings, 253 we conclude that alumina mesh \#600 is an inappropriate pol- 254 ishing medium in the current CBP recipe. For the first time, a 255 large plastically deformed layer produced in a cavity interior sur- 256 face during CBP was demonstrated using metallographic sample 257 preparation. The effect of the rotation speed of the barrel was 258 demonstrated in practice. A simplified scheme for cavity prepa- 259 ration involving both CBP and EP is proposed which is likely 260 
to be competitive with the present industrial cavity preparation procedure.

The authors would like to thank X. Singer and W. Singer for providing the high quality niobium for the current investigation. We are very grateful to our colleagues A. Navitski, A. Ermakov, J. Schaffran for the enormous input for the startup of the current studies. We express our thanks to KEK colleagues and especially Takayuki Saeki and Shigeki Kato for the fabrication of the coupon cavity.

[1] L. Lilje et al., "Improved surface treatment of the superconducting TESLA cavities," Nucl. Instrum. Methods Phys. Res. A, Accel., Spectrom., Detect. Assoc. Equip., vol. 516, no. 2, pp. 213-227, 2004.

[2] Z. Sung, A. Dzyuba, P. Lee, D. Larbalestier, and L. Cooley, "Evidence of incomplete annealing at $800^{\circ} \mathrm{C}$ and the effects of $120^{\circ} \mathrm{C}$ baking on the crystal orientation and the surface superconducting properties of coldworked and chemically polished Nb," Supercond. Sci. Technol., vol. 28, no. 7, 2015, Art. no. 075003 .

[3] H. Kobayashi and M. Matsunaga, "Some experiments on centrifugal barrel finishing," Met Finish, vol. 64, no. 5, pp. 57-60, 1966.

[4] T. Higuchi et al., "Investigation on barrel polishing for superconducting niobium cavities," Proc. 1995 Workshop RF Supercond., Gif-sur-Yvette, France, 1995, pp. 723-727.

[5] T. Higuchi and K. Saito, "Development of hydrogen-free EP and hydrogen absorption phenomena," in Proc. 11th Workshop RF Supercond., Lübek, Germany, 2003, pp. 572-578.

[6] A. D. Palczewski, H. Tian, O. Trofimova, and C. E. Reece, "Detailed surface analysis of incremental centrifugal barrel polishing (CBP) of singlecrystal niobium samples," in Proc. Int. Conf. RF Supercond., Chicago, IL, USA, 2011, pp. 908-911.
[7] A. Palczewski, C. Cooper, B. Bullock, S. Joshi, A. Rossi, and A. Navitski, "R\&D progress in SRF surface preparation with centrifugal barrel polishing (CBP) for both $\mathrm{Nb}$ and $\mathrm{Cu}$," in Proc. Int. Conf. RF Supercond., Paris, France, 2013, pp. 395-400.

[8] C. Cooper and L. Cooley, "Mirror-smooth surfaces and repair of defects in superconducting RF cavities by mechanical polishing," Supercond. Sci. Technol., vol. 26, no. 1, 2012, Art. no. 015011.

[9] A. Palczewski, R. Geng, and H. Tian, "Optimizing Centrifugal barrel polishing for mirror finish SRF cavity and RF tests at Jefferson Lab," in Proc. Int. Particle Accel. Conf., New Orleans, LA, USA, 2012, pp. 24352437.

[10] A. Navitski, E. Elsen, B. Foster, A. Prudnikava, and Y. Tamashevich, 303 "Surface analyses and optimization of centrifugal barrel polishing of $\mathrm{Nb} 304$ cavities," in Proc. Int. Conf. RF Supercond., Whistler, BC, Canada, 2015, 305 pp. 286-290.

[11] S. Lagotzky, G. Müller, A. Navitski, A. Prudnikava, and Y. Tamashevich, 307 "Field emission investigation of centrifugal-barrel-polished NB samples," 308 in Proc. Int. Conf. RF Supercond., Whistler, BC, Canada, 2015, pp. 237- 309 241.

[12] A. Prudnikava, B. Foster, Y. Tamashevich, and A. Ermakov, "R\&D 311 Activities on centrifugal barrel polishing of $1.3 \mathrm{GHz}$ niobium cavities 312 at DESY/University of hamburg," in Proc. Int. Conf. RF Supercond., 313 Lanzhou, China, 2017, Art. no. TUPB111, 4 pages. (in press).

[13] B. Visentin, J. Charrier, B. Coadou, and D. Roudier, "Cavity baking: A 315 cure for the high accelerator field $\mathrm{Q}_{0}$ drop," in Proc. 1999 Workshop RF 316 Supercond., 1999, pp. 198-202.

[14] A. Stierle, T. F. Keller, H. Noei, V. Vonk, and R. Roehlsberger, "DESY 318 NanoLab," J. Large-Scale Res. Facilities JLSRF, vol. 2, no. A76, 2016, 319 pp. 1-6. 320

[15] Y. Tamashevich "Diagnostics and treatment of $1.3 \mathrm{GHz} \mathrm{Nb}$ cavities," 321 Ph.D. dissertation, Dept. Physics, Univ. Hamburg, Hamburg, Germany, 322 2016.

[16] P. Scherrer "Bestimmung der inneren Struktur und der Größe von kol- 324 loidteilchen mittels röntgenstrahlen," Kolloidchemie Ein Lehrbuch, Berlin, 325 Germany: Springer-Verlag, 1912, pp. 387-409 .

[17] G. Williamson, and R. Smallman, "III. Dislocation densities in some 327 annealed and cold-worked metals from measurements on the X-ray debye- 328 scherrer spectrum," Philosoph. Mag., vol. 1, no. 1, pp. 34-46, 1956. 


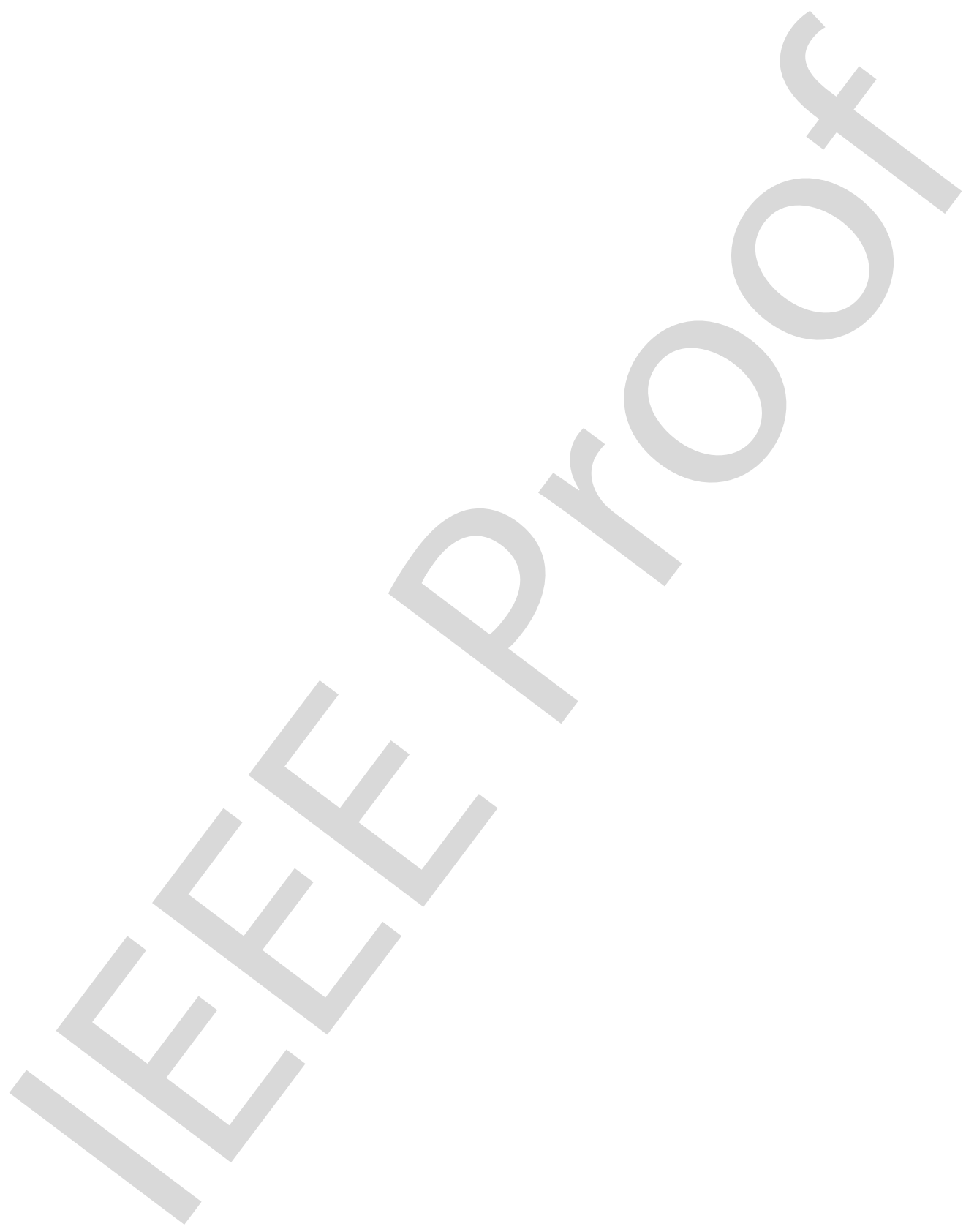

